\title{
Nurturing Plasmonic Properties of Nanocomposite Thin Films: The Importance of Optimum Oblate Shape
}

\author{
Ranjit Laha \\ Department of Physics, Indian Institute of Technology Patna, Bihar-801106, India \\ E-mail: ranjit@iitp.ac.in
}

Received: 30 June 2021; Accepted: 6 August 2021; Available online: 5 October 2021

\begin{abstract}
Metal nanoparticles (MNPs) embedded dielectric thin films are very crucial for many optoelectronic applications. This report investigates various ways of tuning the plasmonic properties of such nanocomposite thin films. For this, the well-known plasmon resonance condition was first generalized to include the shape and volume fraction of MNPs. This was followed by deriving an empirical formula for the resonance position $\left(\lambda_{R}\right)$ which was worked out to be the positive root of a quadratic equation. The coefficients of the deduced quadratic relation involve the parameters obtained from the empirical fit to some of the experimental dielectric functions of MNPs available in literature. The derived working formula enables research community to tune the LSPR of nanocomposites in the whole range of visible wavelengths. The derived formula also concluded that with known lower volume fractions, shape of MNPs affects $\lambda_{\mathrm{R}}$ the most, compared to the other parameters. The derived formula was validated by calculating the full extinction spectra. It was shown for the first time that there exists an optimum value of oblate shape to give maximum resonance for a given nanocomposite.
\end{abstract}

Keywords: Plasmon resonance; Nanocomposite; Geometrical resonance; Gold nanoparticles; Silver nanoparticles.

\section{Introduction}

Noble metal nanoparticles (MNPs) have appealed researchers a lot with respect to the fundamental as well as emerging applied research because of their fascinating optical properties [1-3]. The exciting optical properties appear because of the well-known phenomenon of localized surface plasmon resonance (LSPR) which is the result of coherent oscillation of free electron density at the metal-dielectric interface at the expense of the incident light energy. A peak (fall) in the optical absorbance (transmittance) is the experimental signature of the LSPR occurring in a system. Gold nanoparticles (AuNPs) and silver nanoparticles (AgNPs) are of particular interest, because the LSPR for these metals occur in the visible region of electromagnetic spectrum. The tunable nature of LSPR leads to a large number of applications of AuNPs in the fields of plasmonics, bioscience, sensors and optoelectronics [4-7]. The LSPR positions can become easily tunable in the visible-near infrared region of electromagnetic spectrum if the shape, size, structure and environment of the particles can be controlled. Among the mentioned variations for tunability, controlling environment becomes an easy when the nanoparticles are embedded inside another dielectric matrix. It is even more advantageous for several applications, if the surrounding matrix is transparent the region of LSPR occurrence. Moreover, for some of the plasmonics based optoelectronics applications such as visible photodiode, plasmonic solar cell, and visible light photoconductivity etc., precise control in tuning of plasmonic properties in the visible range is important [7-9].

The well-known Mie theory and its modifications [10-11] explain the optical properties of isolated spherical and ellipsoidal MNPs inside a dielectric medium. As per Mie theory, the extinction cross section $\left(\sigma_{\text {ext }}\right)$ of the dipole mode of oscillation is given by

$$
\sigma_{e x t}=\frac{24 \pi^{2} \varepsilon_{d}{ }^{3 / 2} R^{3}}{\lambda}\left[\frac{\varepsilon_{2}}{\left(\varepsilon_{1}+2 \varepsilon_{d}\right)^{2}+\varepsilon_{2}{ }^{2}}\right]
$$

where, $\varepsilon_{1}$ and $\varepsilon_{2}$ are respectively the real and imaginary parts of the frequency dependent dielectric function of the metal, $\varepsilon_{\mathrm{d}}$ is the dielectric constant of the surrounding medium and $\mathrm{R}$ is the radius of the nanoparticle. The spectral position of LSPR is governed by the well-known plasmon resonance condition which can be deduced from Eq. 1 as

$$
\varepsilon_{1}=-2 \varepsilon_{d}
$$


Equation 2 is also known as Fröhlich condition (FC) [12]. The wavelength at which Eq. 2 is satisfied is the LSPR position $\left(\lambda_{R}\right)$. The factor of 2 in Eq. 2 is for spherical shape and takes different real and positive values other than 2 for non-spherical shapes. It may be noted that Eq. 2 has only shape and material dependence, pointing out that $\lambda_{R}$ does not change with size. Nevertheless, the size contributes to the strength of resonance through the $R^{3} / \lambda$ term in Eq. 1. There are many experimental reports on tuning the plasmonic positions including for the cases of nanostar, nanocube, nanorod, and AuNP-polymer composite etc. [13-18]. Even theoretical simulations have been performed by Eremina et al [19] for the nanoparticles of oblate shapes supported on different substrates, showing the variation of $\lambda_{\mathrm{R}}$ with different aspect ratios. Recently, Sun et al performed similar experiment and theoretical calculations for Au-Ag alloy nanobars [20]. Almost all the tuning related works reported so far in the literature revolve around the variation of $\lambda_{\mathrm{R}}$ with respect to the variation in shape and surrounding dielectric medium as per Eq. 2. Despite of many known important consequences of tuning, any direct working formula for $\lambda_{R}$ is not found in literature for embedded MNPs, particularly, in the case of thin film nanocomposites. With the emergence of new and highly controllable fabrication schemes [21-22], such working formula turns out to be even more crucial need of the hour.

The present work revisits the resonance condition for nanocomposite thin films so as to include the shape and volume fraction, and for the first time (ii) derives a working formula that helps in tuning the plasmonic position and (ii) demonstrates the existence of an optimum shape for maximum optical density for a given pair of metal nanoparticle-dielectric film combination.

\section{Theoretical Details}

When light passes through a medium containing small particles, the transmitted intensity reduces along the distance of the medium as a result of scattering and absorption of light by the particles, and is given by

$$
I=I_{0} \exp \left(-n \sigma_{e x t} z\right)
$$

The quantity $n \sigma_{e x t} z$ is also known as the optical density of the medium, which depends on the distance (z) travelled by light inside the medium, the number density $(n)$ of the particles in the medium and the extinction cross section $\left(\sigma_{\text {ext }}\right)$ of a single particle. The extinction cross section contains the contributions from both absorption and scattering through the linear relation

$$
\sigma_{e x t}=\sigma_{a b s}+\sigma_{s c a}
$$

where $\sigma_{a b s}$ and $\sigma_{s c a}$ are respectively the absorption and scattering cross sections of the particle. The extinction cross section can be calculated under quasi-static approximation, using the polarizability $(\alpha)$ as

$$
\begin{gathered}
\sigma_{a b s}(\lambda)=\frac{4 \pi^{2}}{\lambda} \operatorname{Im}\{\alpha(\lambda)\} \\
\sigma_{s c a}(\lambda)=\frac{128 \pi^{6}}{\lambda^{4}}|\alpha(\lambda)|^{2}
\end{gathered}
$$

with ' $\operatorname{Im}\{\alpha(\lambda)\}$ ' being the imaginary part of $\alpha(\lambda)$. Equations 3-5 indicate that the incident light energy is used in polarizing the particle of the medium. The polarizability of the particle depends on the material properties of both particle and the surrounding, size and shape of the particle and the incident light wavelength. The general expression for the diagonal elements of the polarizability tensor for an ellipsoidal particle with $a, b, c$ as the major axes can be given by [12]

$$
\alpha_{j j}=\frac{4 \pi a b c}{3} \frac{\varepsilon-\varepsilon_{d}}{L_{j}\left(\varepsilon-\varepsilon_{d}\right)+\varepsilon_{d}}
$$

The frequency dependent dielectric function of the metallic particle, in general a complex quantity, is expressed as

$$
\varepsilon=\varepsilon_{1}+i \varepsilon_{2}
$$

$L_{i}$, called as the depolarization factor along the $i^{\text {th }}$ axis of the ellipsoid, is a function of ratio of axes such that 


$$
\sum_{i=1}^{3} L_{i}=1
$$

For a sphere $L_{1}=L_{2}=L_{3}=1 / 3$.

Looking at the rarity of both theoretical and experimental studies on the general case of ellipsoidal particles, the present discussion is limited to spheroids only. Spheroid, also known as the ellipsoid of rotation, is a special case of an ellipsoid when two of the axes of the ellipsoid are equal. A spheroid can be either a prolate $(a=b<c)$ or an oblate $(a=b>c)$. Thus, for a spheroid, $L_{1}=L_{2}=L$ and $L_{3}=L_{\mathrm{z}}$. The functional dependences of $L$ on the axes dimensions are different for oblate and prolate. In the case of thin film nanocomposites, only $L$ is active $\left(L_{z}\right.$ falls in the direction of incident light), and is given by [23]

$$
L=\frac{e}{4 q^{3}}\left[\pi-2 e q-2 \operatorname{arctg} \frac{e}{q}\right]
$$

where, $q=\sqrt{1-e^{2}}$ with $e(<1)=$ height $/$ Diameter $=h / 2 R$ and $R$ is the in plane radius of the oblate. Finally, the polarizability of an oblate can be expressed as

$$
\alpha=\frac{4 \pi R^{2} h(K+1)}{3} \frac{\varepsilon-\varepsilon_{d}}{\varepsilon+K \varepsilon_{d}}
$$

Here, $K$ is the shape factor, given by

$$
K=\frac{1}{L}-1
$$

Thus, for a sphere, $\mathrm{K}=2$.

The frequency or wavelength dependency of $\sigma_{\text {ext }}$ comes from the dielectric function of the MNPs. After using Eq. 9 in Eq. 5, the condition for the maximum extinction, which is also known as the resonance condition, can be written as

$$
\varepsilon_{1 R}=-K \varepsilon_{d}
$$

It is clear from Eq. 12 that since both $\mathrm{K}$ and $\varepsilon_{d}$ are positive, the embedded particles need to be of some metal for a resonance to occur. With $K=2$, Eq. 12 reduces to the well-known FC for isolated spheres in a medium. The resonance condition given by Eq. 12 is only a relation among the dielectric function and shape of the MNPs, and the surrounding dielectric constant. Equation 12, however, can be used to arrive at the resonance condition $\lambda_{R}$ if the expressions for $\varepsilon_{1}$ and $\varepsilon_{2}$ as a function of wavelength are used. For this, the common practice is to use eithr the Drude free electron model or the Lorentz-Drude model both of which provide the frequency dependent dielectric function of metals [24]. This practice has also been adopted for metal-dielectric nanocomposites earlier [25]. However, many experimentally determined dielectric function data deviate substantially from the free electron based theoretical models. The main reason of the deviation seems to be the quality and dimension (bulk/thin film/thick film/nanoparticles) of the material, originated from the method preparation. Therefore, proper modeling of LSPR positions requires the consideration of the practical values. Hence, some of the reported experimental data were fitted in the range 500-900 nm and 400-800 $\mathrm{nm}$ to find $\varepsilon_{1}(\lambda)$ for Au and Ag respectively. From the fit, the functional dependency of $\varepsilon_{1}(\lambda)$ was found to be $\varepsilon_{1}(\lambda)=a-b \lambda-c \lambda^{2}$, where the values of $a, b$ and $c$ are as summarized in Table 1 for the two sets of data each for Au and Ag. The raw data sets were taken from a free downloadable database.

Table 1. Fitting details for finding $\varepsilon_{1}(\lambda)$ of published experimental data of Au and Ag.

\begin{tabular}{lllllll}
\hline & $\mathrm{Au}$ & \multicolumn{5}{c}{$\mathrm{Ag}$} \\
\cline { 2 - 7 } & $\mathrm{a}$ & $\mathrm{b}$ & $\mathrm{c}$ & $\mathrm{a}$ & $\mathrm{b}$ & $\mathrm{c}$ \\
\hline Emprical 1 $^{*}$ & 21.6 & $3.4 \times 10^{-2}$ & $2.9 \times 10^{-5}$ & 8.5 & $1.6 \times 10^{-2}$ & $4.2 \times 10^{-5}$ \\
Emprical 2 $^{* *}$ & 19.1 & $2.6 \times 10^{-2}$ & $3.5 \times 10^{-5}$ & 6.1 & $5.5 \times 10^{-3}$ & $4.5 \times 10^{-5}$ \\
\hline
\end{tabular}

*Ref-26, ** Ref-27 \& 28 for Au and Ag respectively.

It is clear from Table 1 that even though the orders of magnitude are same, the actual values of the co-efficients vary for the different experimental data. This reiterates the fact that experimental conditions cannot be neglected while modelling the plasmonic properties. For example, the Johnson and Christy (JC) data provides the dielectric 
function for micrometer thick films whereas the data reported by Rosenblatt (RB) and Ciesielski (AC) give that for thin films below $100 \mathrm{~nm}$ [26-28]. Using Eq. 12 in the above fitted equation, the quadratic equation involving $\lambda_{\mathrm{R}}$ becomes

$$
c \lambda_{R}^{2}+b \lambda_{R}-\left(a+K \varepsilon_{d}\right)=0
$$

It is clear that both the roots of Eq. 13 are real. Considering the physical significance, the positive root of Eq. 13 gives the empirical working formula for resonant wavelength as

$$
\lambda_{R}=\frac{\sqrt{b^{2}+4 c\left(a+K \varepsilon_{d}\right)}-b}{2 c}
$$

The models for isolated particles as mentioned in Eq. 12 do not work for the samples having embedded aggregates of MNPs. Additionally, LSPR peak features dramatically deviate for aggregates involving MNPs with non-spherical shapes, when compared to the spherical counterparts with equivalent volume. Effective medium theories are used to explain the optical properties of such systems. Dynamical Maxwell-Garnet (DMG) theory [29] is one such effective medium theory that takes the non-spherical shape into account while explaining the optical properties of aggregates of MNPs under the assumption of low volume fraction of MNPs. As per DMG theory, the effective dielectric function $\left(\varepsilon_{e f f}\right)$ of a two phase composite system is given by

$$
\varepsilon_{e f f}=\varepsilon_{d} \frac{\varepsilon+K \varepsilon_{d}+f K\left(\varepsilon-\varepsilon_{d}\right)}{\varepsilon+K \varepsilon_{d}-f\left(\varepsilon-\varepsilon_{d}\right)}
$$

Equation 15 helps to find the resonance condition in the following way. In general, $\varepsilon_{\text {eff }}$ is complex (through $\varepsilon$ ) whose real and imaginary parts are respectively responsible for scattering and absorption. Since the optical density is directly proportional to the extinction cross-section, resonance condition in optical density can be characterized as the condition for maxima in $\left|\varepsilon_{\text {eff }}\right|$. The resonance condition, thus, can be obtained as

$$
\varepsilon_{1}=-\left(\frac{K+f}{1-f}\right) \varepsilon_{d}
$$

Equation 16 is the generalized plasmon resonance condition that involves both volume fraction and shape of the nanoparticles. Since K can have 3 different values as mentioned in Eq. 9, there can be three different instances of the resonance condition being satisfied for arbitrary suspension of ellipsoids in a medium. It can be easily seen that Eq. 16 reduces to Eq. 12 when the volume fraction approaches zero. Another important aspect of the generalized resonance condition is realized in the following way. The FC predicts that the resonance position red shifts with increase in the $\varepsilon_{\mathrm{d}}$. The corresponding shape dependent variant (Eq. 12) additionally predicts that resonance position red shifts with the shape becoming more and more away from the spherical shape for a given pair of metal-dielectric combination. However, both of the earlier expressions predicted no effect on the LSPR position upon changing the concentration of nanoparticles by either changing the size or number density or both of the nanoparticles. In fact, the volume of the nanoparticle is included in the polarizability of the particle, and acts just as a scaling factor for the resonance strength. The generalized resonance condition given in Eq. 16 indicates that LSPR position also changes with volume fraction. After including the generalized resonance condition, the working formula for tuning the LSPR position becomes

$$
\lambda_{R}=\frac{\sqrt{b^{2}+4 c(a+\psi)}-b}{2 c}
$$

With

$$
\psi=\left(\frac{K+f}{1-f}\right) \varepsilon_{d}
$$

\section{Numerical results and discussion}

Figure 1 represents the surface plots showing the variation of LSPR position with volume fraction and shape factor for both AuNPs and AgNPs embedded in media that are recognized with different values of dielectric 
constants. The media chosen for the analysis here are silica glass $\left(\mathrm{SiO}_{2}, \varepsilon_{\mathrm{d}}=2.25\right)$ alumina $\left(\mathrm{Al}_{2} \mathrm{O}_{3}, \varepsilon_{\mathrm{d}}=3.14\right)$ indium tin oxide (ITO, $\left.\varepsilon_{\mathrm{d}}=4\right)$. It is to note here that all the media chosen here represent some of the materials that are useful in many practical applications. For the sake of theoretical comparison the calculations were also performed for air as one of the media and are given Fig. 1. However, it is noteworthy that MNPs embedded air is not a practically feasible system, as nanoparticles cannot be suspended in air without the support of a substrate of $\varepsilon_{d}>1$. As can be seen from Fig. 1, changing the medium shifts the whole surface even for the lowest values of $f$ and $K$. The vertical separations at $K=10$ and $f=0$ are $375 \mathrm{~nm}$ and $400 \mathrm{~nm}$ respectively for AuNPs and AgNPs, when the embedding media are changed from air to ITO. The corresponding values at $K=2$ and $f=30 \%$ are $\sim 145 \mathrm{~nm}$ and $180 \mathrm{~nm}$ respectively for AuNPs and AgNPs. This indicates that changing embedding medium has more effect on higher $K$ values for a given $f$ value. The effect is highest, as expected, at $K=10$ and $f=30 \%$. Thus, for a unit change in $\varepsilon_{\mathrm{d}}$, the LSPR position changes by 40-80 nm. The importance of this outcome of Fig. 1 lies in the fact that in most of the practical situations, $\varepsilon_{\mathrm{d}}$ can easily change by a fraction of unity even from one batch to another during the growth thereby changing the LSPR position by $~ 5-10 \mathrm{~nm}$ from the intended value. Hence, observation of such changes in $\lambda_{\mathrm{R}}$ is very common for nanocomposites formed with metal oxides. When the individual changes in the LSPR positions of AuNPs and AgNPs are compared, all the trends look similar except for (i) the absolute values of the LSPR positions and (ii) the variations relative to a particular parameter are slightly higher in AgNPs. While the first exception can be understood to be due to the different values of $\varepsilon_{1}$ at same wavelength for $\mathrm{Au}$ and $\mathrm{Ag}$ as reflected in Table 1, the second exception was further analyzed elaborately as shown in Fig. 2. Figure 2a and b show the relative changes in $\lambda_{\mathrm{R}}$ for unit change in $K$ for the media glass and ITO. Similarly, Fig. 2c and d show the corresponding values for every $1 \%$ change in the volume fraction. It was found using the expression for $K$ that changing $K$ by one unit involves decreasing the height by $5-6 \mathrm{~nm}$ for an oblate with in-plane radius of $25 \mathrm{~nm}$. It is clear that for unit change in K, LSPR position changes by 30-60 nm for AuNPs and 30-80 nm for AgNPs. Similarly, for every $1 \%$ change in $f$, LSPR position changes by 1-5 $\mathrm{nm}$. Another noteworthy observation is that sensitivity of LSPR decreases with increase in $K$ but shows reverse trend with $f$. The combined analysis of the derived working formula through Figs. 1 and 2 indicate that among the three parameters discussed here, changing shape has the maximum effect on shift in LSPR position.

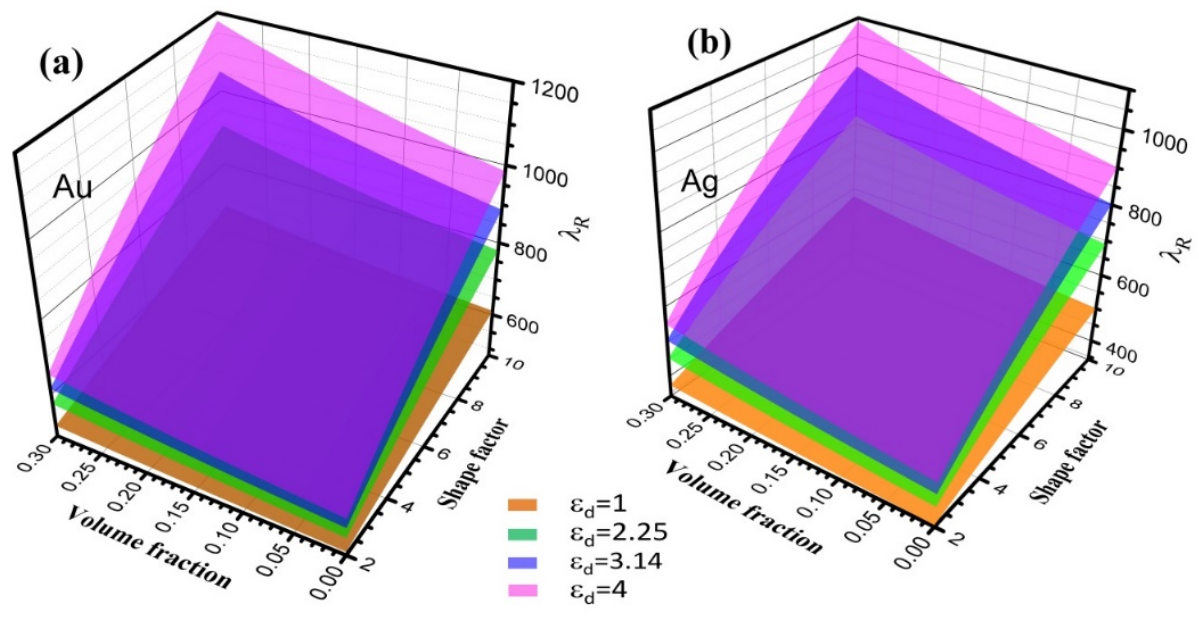

Fig. 1. Variation of LSPR with volume fraction and shape factor for AuNPs and AgNPs embedded in different media.

Figure 3 shows the extinction cross-section $\left(\sigma_{\text {ext }}\right)$ of the isolated AuNPs embedded in different media, calculated by making use of Eq. 9 followed by using Eqs. 2-4.The radius of the oblate used during the calculation was $25 \mathrm{~nm}$. Calculations for cross-section were initially performed with different dielectric function data. However, no substantial change was observed with varying the empirical dielectric function data. Therefore, for all the results shown here, only JC data has been used. As can be seen in Fig. 3, both the position and intensity of $\sigma_{\text {ext }}$ varies with changing $K$ and $\varepsilon_{\mathrm{d}}$. When the radius was varied, keeping the shape fixed, only overall intensity was found to change with no change in position. For a given medium, position of $\sigma_{\text {ext }}$ keeps on increasing with increase in $K$. However, the trend for the resonant value of $\sigma_{\text {ext }}\left(\sigma_{R}\right)$ shows an initial increase followed by decrease in the wavelength range shown here. Similar variation in $\sigma_{\mathrm{R}}$ with $K$ was also observed in the case of AgNPs embedded inside different media as shown in Fig. 4. It may be noted as shown in both Figs. 3 and 4 that beyond certain values of K, the LSPR position does not remain within the visible range. For AuNPs inside ITO, the maximum value of $\mathrm{K}$ to get a resonance in the visible region is $~ 7$. Similarly, for AuNPs inside air, SiO2, and Al2O3, the maximum values of 
K are respectively 25, 12 and 9. The corresponding values for AgNPs inside air, SiO2, Al2O3 and ITO are 40, 12, 9 and 7 respectively. When the spectra for AuNPs and AgNPs are compared, the first observation is the difference in the overall value of $\sigma \mathrm{R}$ which is more than one order of magnitude higher for AgNPs for the same set of parameters.
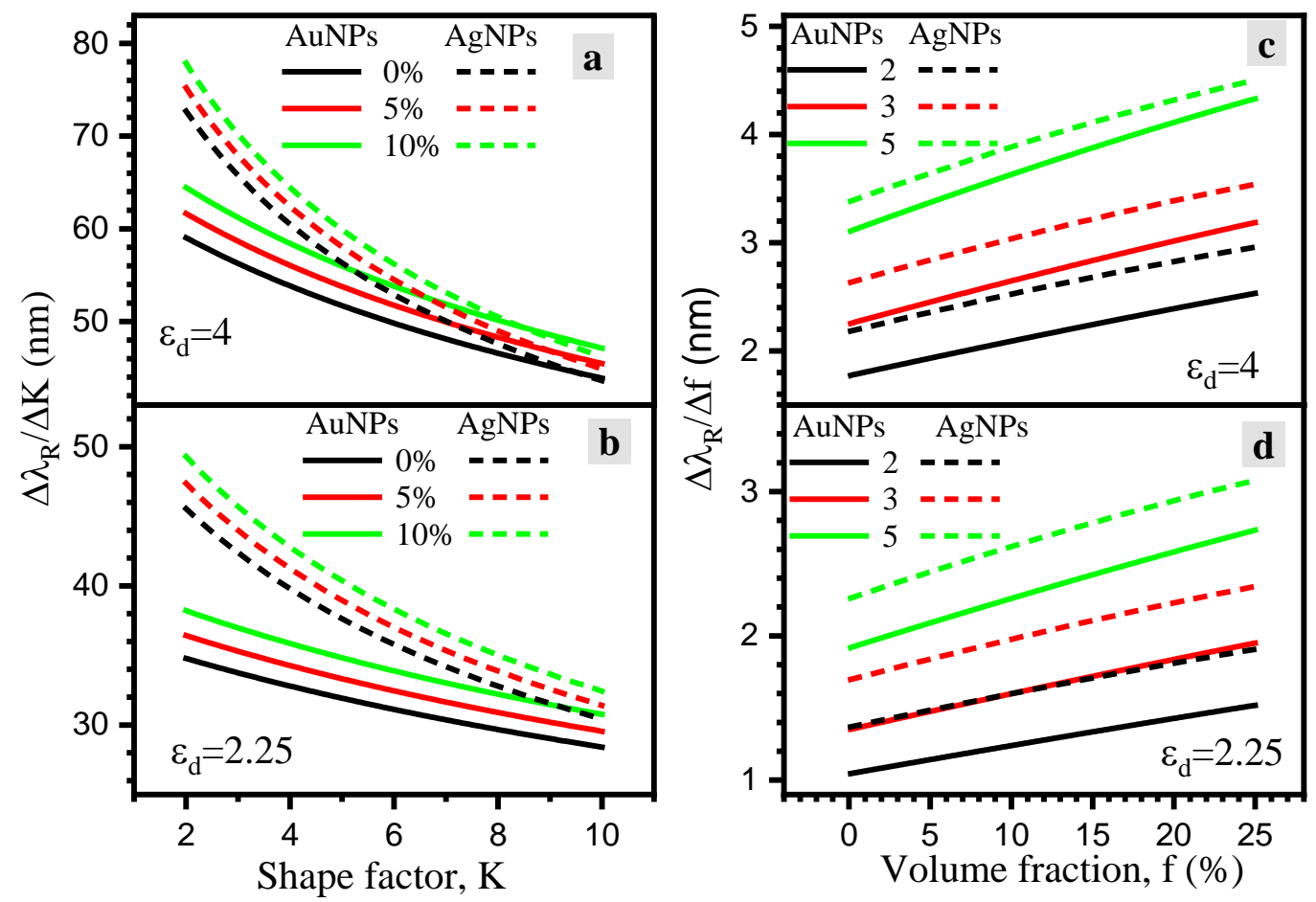

Fig. 2. Relative changes in $\lambda_{\mathrm{R}}$ for unit change in $K(\mathbf{a} \& \mathbf{b})$ and for every $1 \%$ change in the volume fraction (c $\&$ d) for the media glass and ITO.

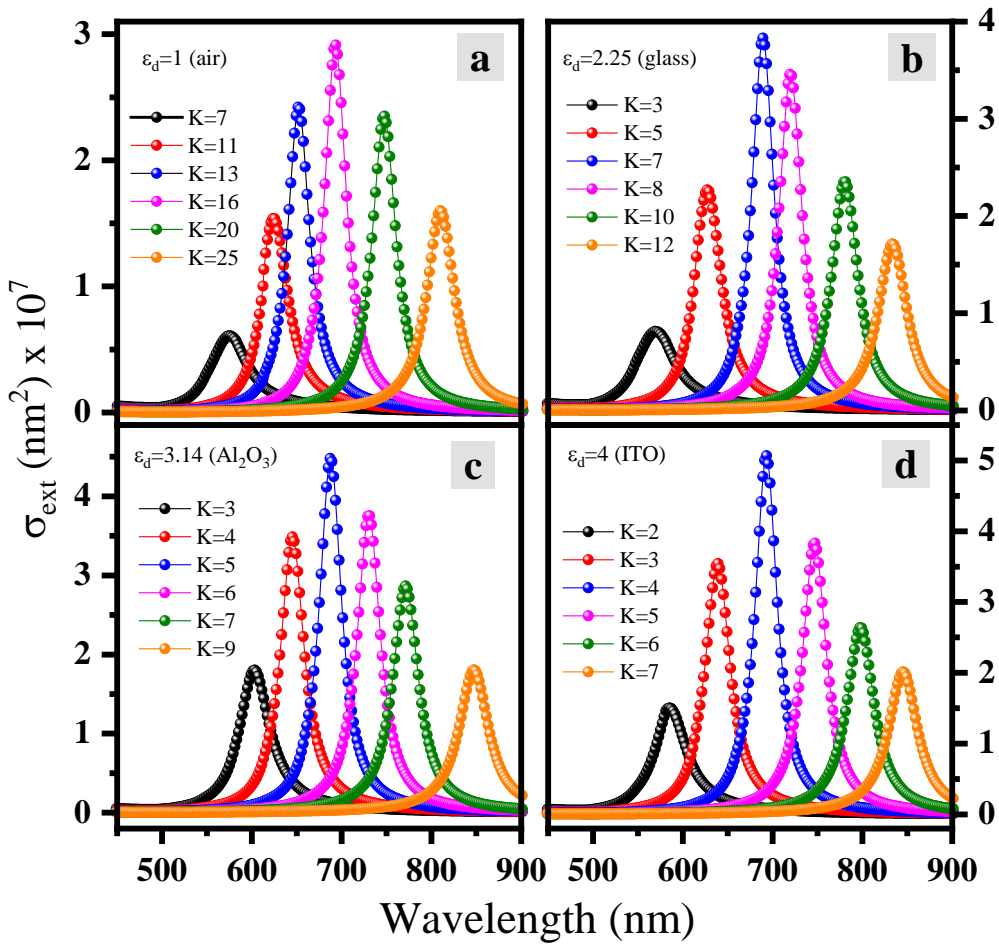

Fig. 3. The calculated extinction cross-section $\left(\sigma_{\text {ext }}\right)$ of the isolated AuNPs embedded in different media. 


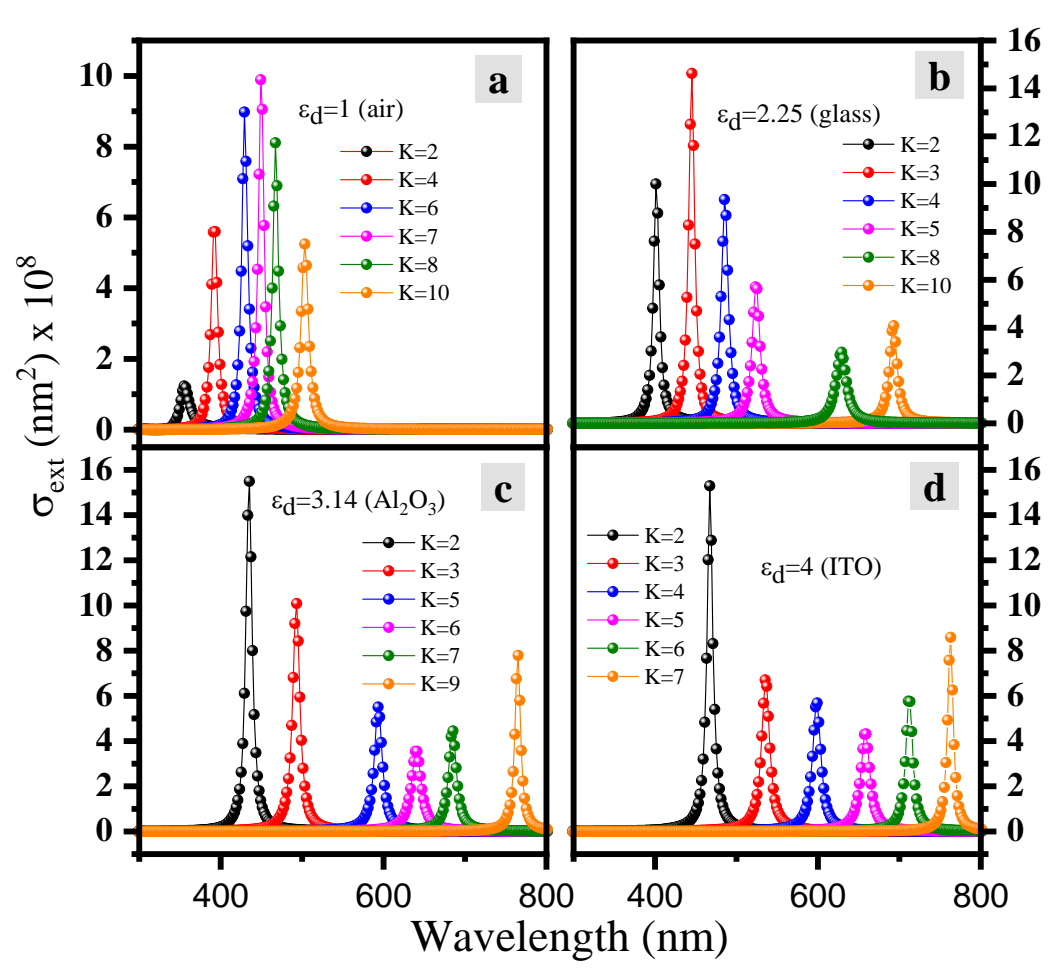

Fig. 4. The calculated extinction cross-section $\left(\sigma_{\mathrm{ext}}\right)$ of the isolated AgNPs embedded in different media.

The most important difference between the calculated extinction cross sections for Au and Ag appears in the form of the width of the peaks. The full width at half maxima (FWHM) is smaller in case of AgNPs. Also, the trend in variation of $\sigma_{\mathrm{R}}$ with $K$ is different compared to that in the case of AuNPs. Figure 5 shows such trends separately for different media. In Fig. 5, only $\sigma_{\mathrm{R}}$ values have been plotted against $K$. The optimum $K$ values $\left(K_{R}\right)$ showing the maximum strength of resonance are also mentioned in each panel along with the corresponding aspect ratio of the oblate $(\mathrm{h} / \mathrm{R})$. The value of $\mathrm{KR}$ decreases with increasing $\varepsilon \mathrm{d}$ for AuNPs. However, there appears to be two such optimum values for each media in case of AgNPs. For media with high $\varepsilon d$, the second one falls in the visible range. Whereas the values of $\mathrm{K}$ indicated in Fig. 5e and $\mathrm{f}$ are the second optimum ones, the value indicated in Fig. $5 g$ is the first optimum. For AgNPs in air, the second optimum does not fall in the range of K-values shown here. In addition to the analysis of the resonant maximum vs $\mathrm{K}$, the $\lambda \mathrm{R}$ vs $\mathrm{K}$ was also compared with the same plotted from the derived working formula. Figure 6 shows the comparison. In Fig. 6 a and b, the symbols represent the positions obtained from the full calculation using the JC data, solid lines represent the positions obtained using the derived working formula (Eq. 17) with JC data. Similarly, in Fig. 6c and d, the comparison of the positions calculated using the two sets of dielectric functions each for AuNPs and AgNPs as given in Table 1 are shown. Figure 6validates the derived working formula for both AuNPs and AgNPs, as the values obtained from full spectra calculation match very well with the LSPR vs K obtained using the derived formula. The small deviation observed for the higher dielectric constant media may be due to the difference between fitted and actual values of $\varepsilon 1$. This also indicates that even though the fitting was done for a small range of 400-900 nm, the derived working formula works well even at higher wavelengths, as the LSPR position for high K values goes well beyond $900 \mathrm{~nm}$. When different dielectric functions of AuNPs are considered, as shown in Fig. 6c, LSPR positions vary negligibly. However, in case of AgNPs, the positions obtained using the data by AC are higher than those obtained using the JC data. The reason for this could be the lower thickness and the annealing conditions used to form the AgNPs in case of AC data. Nevertheless, the derived working formula enables one to tune the plasmonic position with desired level of control in the entire range of visible region.

In order to further probe in knowing the mechanism behind the existence of the optimum shape, expression for the polarizability was re-written as

$$
\begin{aligned}
\alpha & =\beta(K, R) \Lambda\left(\varepsilon, \varepsilon_{d}, K\right) \\
\beta & =\frac{4 \pi R^{2} h(K+1)}{3} \\
\Lambda & =\frac{\varepsilon-\varepsilon_{d}}{\varepsilon+K \varepsilon_{d}}
\end{aligned}
$$




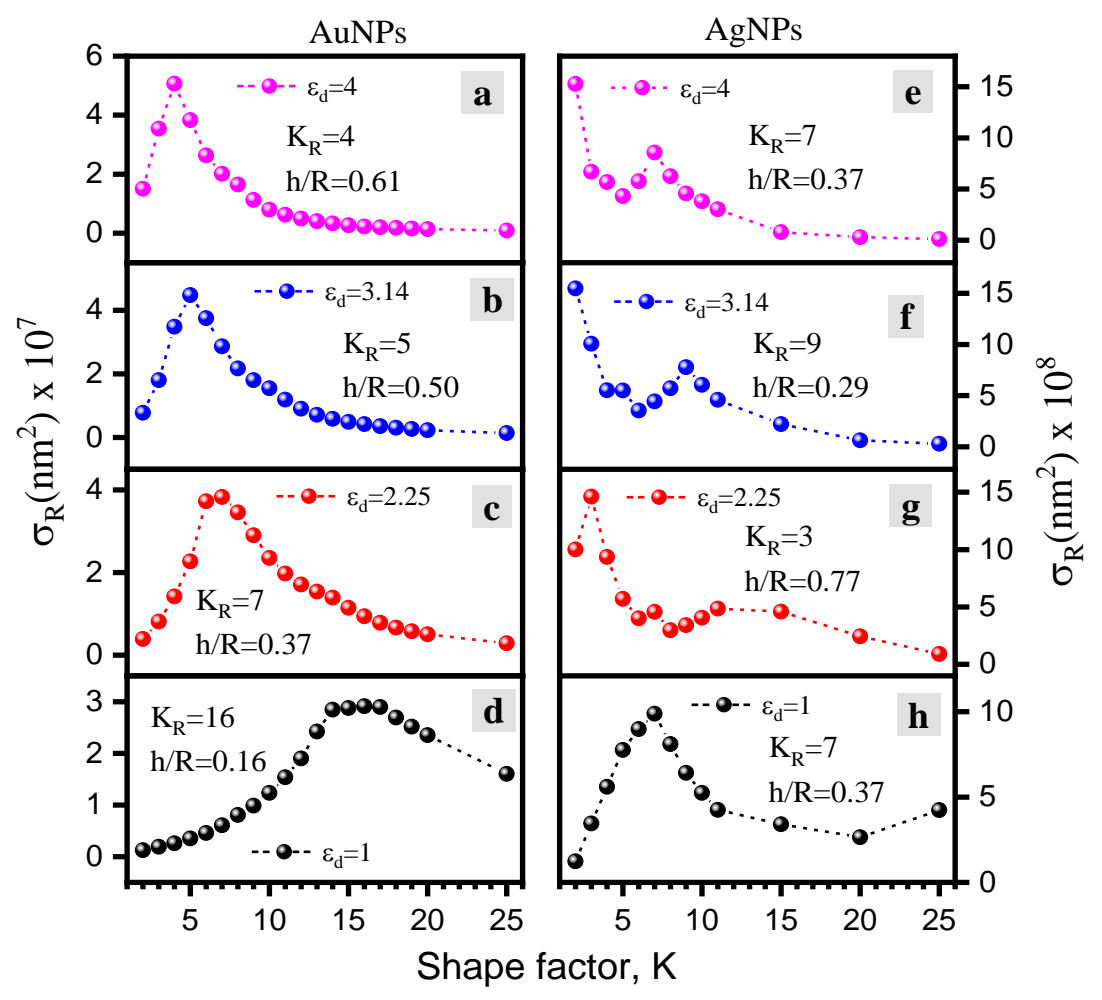

Fig. 5. Variation of the resonant value $\left(\sigma_{\mathrm{R}}\right)$ of extinction cross-section with shape factor showing the optimum shape factor of oblate for maximum resonance in different media. The corresponding optimum aspect ratio $(\mathrm{h} / 2 \mathrm{R})$ is also indicated in panel.

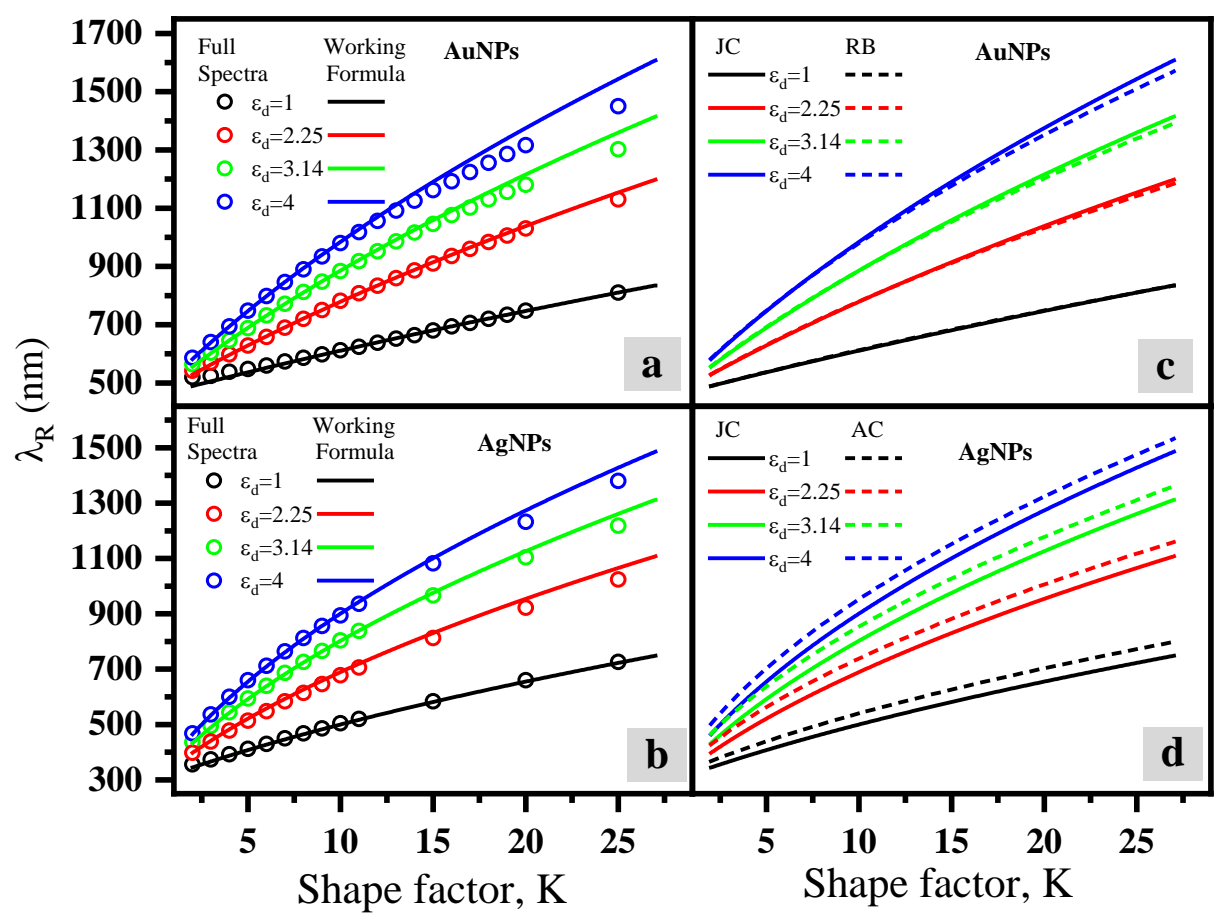

Fig. 6. Validation of the working formula by comparison with ' $\lambda_{R}$ vs $K$ ' obtained from full spectra (a \& b), and comparison of ' $\lambda_{\mathrm{R}}$ vs K' with two dielectric functions each for AuNPs and AgNPs (c \& d).

Wherein, the factor $\beta(K)$ does not contain any material information unlike $\Lambda\left(\varepsilon, \varepsilon_{\mathrm{d}}, K\right)$ which contains both the material properties (through $\varepsilon$ and $\varepsilon_{\mathrm{d}}$ ) and geometry (through $K$ ). It is noteworthy that one of the major contributors in deciding the value of $\sigma_{\mathrm{R}}$ is the term (volume) $)^{2} / \lambda^{4}$ as given in Eq. 4-5. Thus, it is clear that major role of $\beta$ is to 
decide the strength of resonance (the value of $\sigma_{\mathrm{R}}$ ), as $\Lambda$ mainly decides the position of resonance. At first glance, the form of $\beta(K)$ gives an impression that it varies linearly with $K$ irrespective of the volume of the particle. However, variation of shape keeping the volume fixed need not be an ideal situation always. When the in-plane radius $(R)$ is kept constant, the value of $h$ automatically gets changed when $K$ is changed. Therefore, in order to know how $\beta$ changes with $K$, it is important to know how $h$ changes with $K$. Figure 7 shows the plot of $\beta$ vs $K$. The observation from Fig. 7 clearly indicates that there exists a geometry originated resonance which is independent of the material properties $\left(\varepsilon_{\mathrm{d}}\right.$ and $\varepsilon$ ) and purely depends on the geometry of the particle relative to the incident light direction. What is observed in the final extinction spectrum is the superposition of the geometrical resonance given by $\beta$ and the plasmon resonance (material property) given by $\Lambda$. When only a sphere or a single geometry is considered, the geometrical resonance does not come into picture, resulting in appearance of size related variation in the strength of resonance at a fixed wavelength or frequency. When shape is varied, the resonance strength varies having geometry originated resonance. Thus, maximum utilization of a given volume of the plasmonic nanoparticle involves forming a shape that has the optimum ratio of the dimensions in the direction perpendicular to the direction of the incident light relative to the dimension along the direction of light.

It is important to mention the possible deviations which may appear while making practical use of the derived formula. Deviations may appear if (i) the desired LSPR position is beyond $900 \mathrm{~nm}$, (ii) proper value of $\varepsilon_{\mathrm{d}}$ is not known/used, (iii) the size is so small that quantum size effect starts appearing in the optical properties, particularly in case of AgNPs [30], and (iv) Volume fraction of the metal nanoparticle is high enough to defy the basic assumption of no interparticle coupling.

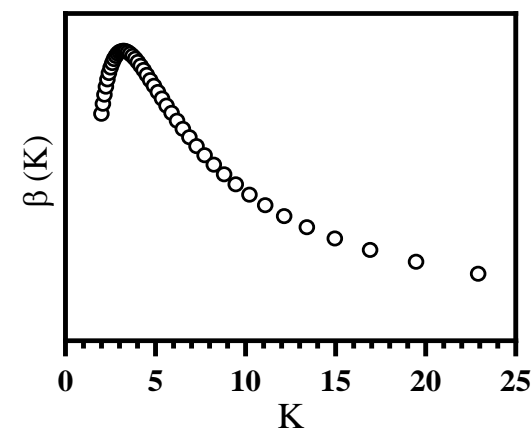

Fig. 7. Geometrical resonance in oblate shape $(\mathrm{R}=25 \mathrm{~nm})$, independent of material properties of the nanocomposite.

\section{Conclusion}

The plasmon resonance condition was generalized to include the volume fraction and shape of MNPs. Using some of the published dielectric function data, a working formula was derived to show different ways of tuning the plasmonic properties of metal-dielectric nanocomposites designed by embedding MNPs inside a dielectric thin film. From the analysis of the derived relation, it was found that LSPR of nanocomposites can be varied in the entire visible range. The analysis also showed that among the three parameters discussed here, changing shape has the maximum effect on shifting the LSPR position. Based on quasi-static theory, the shape dependent extinction spectra were calculated for the oblate shaped AuNPs/AgNPs embedded in different media. The analysis of the extinction spectra showed that there exists an optimum shape of oblate to give maximum strength of resonance. The reason behind the existence of such optimum shape was attributed to the contribution of geometrical resonance to the extinction cross-section.

\section{References}

[1] Cheng O, Son D, Sheldon M. Light-induced magnetism in plasmonic gold nanoparticles. Nature Photonics. 2020; 14(6):365-368.

[2] Mark I Stockman et al, Roadmap on plasmonics. Journal of Optics. 2018; 20: 043001.

[3] Ugwuoke L, Mančal T, Krüger T. Localized Surface Plasmon Resonances of Simple Tunable Plasmonic Nanostructures. Plasmonics. 2019; 15(1):189-200.

[4] Tong L, Wei Q, Wei A, Cheng J. Gold Nanorods as Contrast Agents for Biological Imaging: Optical Properties, Surface Conjugation and Photothermal Effects. Photochemistry and Photobiology. 2009; 85(1):21-32.

[5] Pan J, Chen J, Zhao D, Huang Q, Khan Q, Liu X et al. Surface plasmon-enhanced quantum dot light-emitting diodes by incorporating gold nanoparticles. Optics Express. 2015; 24(2):A33. 
[6] Baliyan A, Usha S, Gupta B, Gupta R, Sharma E. Localized surface plasmon resonance-based fiber-optic sensor for the detection of triacylglycerides using silver nanoparticles. Journal of Biomedical Optics. 2017; 22(10):1.

[7] Kumara N, Chau Y, Huang J, Huang H, Lin C, Chiang H. Plasmonic spectrum on 1D and 2D periodic arrays of rod-shape metal nanoparticle pairs with different core patterns for biosensor and solar cell applications. Journal of Optics. 2016; 18(11):115003.

[8] Shokeen P, Jain A, Kapoor A. Embedded vertical dual of silver nanoparticles for improved $\mathrm{ZnO} / \mathrm{Si}$ heterojunction solar cells. Journal of Nanophotonics. 2017; 11(04):1.

[9] Sekhar M, Kumar R, Kumari S, Laha R, Pandey S, Kar M. Visible light photoconductivity studies of gold nanoparticle embedded $\mathrm{ZnO}$ thin films for photo detector application. Semiconductor Science and Technology. 2020; 35(11):115004.

[10] Mie G. Contributions on the optics of turbid media, particularly colloidal metal solutions. Annalen der Physik, 1908; 25:377-445.

[11] Gans R. About the shape of ultra-microscopic gold particles. Annals of Physics. 1912;342(5):881-900. (in German)

[12] Bohren C, Huffman D. Absorption and scattering of light by small particles. New York: Wiley; 2013.

[13] Barbosa S, Agrawal A, Rodríguez-Lorenzo L, Pastoriza-Santos I, Alvarez-Puebla R, Kornowski A et al. Tuning Size and Sensing Properties in Colloidal Gold Nanostars. Langmuir. 2010;26(18):14943-14950.

[14] Sun Y. Shape-Controlled Synthesis of Gold and Silver Nanoparticles. Science. 2002;298(5601):2176-2179.

[15] Nikoobakht B, El-Sayed M. Preparation and Growth Mechanism of Gold Nanorods (NRs) Using SeedMediated Growth Method. Chemistry of Materials. 2003;15(10):1957-1962.

[16] Batra D, Seifert S, Varela L, Liu A, Firestone M. Solvent-Mediated Plasmon Tuning in a Gold-NanoparticlePoly (Ionic Liquid) Composite. Advanced Functional Materials. 2007;17(8):1279-1287.

[17] Truong P, Ma X, Sim S. Resonant Rayleigh light scattering of single Au nanoparticles with different sizes and shapes. Nanoscale. 2014; 6(4):2307.

[18] Kamakshi K, Sekhar K, Almeida A, Agostinho Moreira J, Gomes M. Tuning the surface plasmon resonance and surface-enhanced Raman scattering of pulsed laser deposited silver nanoparticle films by ambience and deposition temperature. Journal of Optics. 2014; 16(5):055002.

[19] Eremina E., Y. Eremin, W. Thomas. Discrete sources method for simulation of resonance spectra of nonspherical nanoparticles on a plane surface. Optics Communication. 2005; 246:405-413.

[20] Sun F, Du C, Fu T, Chen Y, Sun L, Zhang R et al. Optimal aspect ratio and excitation spectral region of individual Au Ag1- alloy nanobars for plasmonic sensing. Physics Letters A. 2020;384(30): 126785.

[21] Salley D, Keenan G, Grizou J, Sharma A, Martín S, Cronin L. A nanomaterials discovery robot for the Darwinian evolution of shape programmable gold nanoparticles. Nature Communications. 2020;11(1).

[22] Desireddy A, Conn B, Guo J, Yoon B, Barnett R, Monahan B et al. Ultrastable silver nanoparticles. Nature. 2013;501(7467):399-402.

[23] Giordano S. Effective medium theory for dispersions of dielectric ellipsoids. Journal of Electrostatics. 2003;58(1-2):59-76.

[24] Rakić A, Djurišić A, Elazar J, Majewski M. Optical properties of metallic films for vertical-cavity optoelectronic devices. Applied Optics. 1998; 37(22):5271.

[25] Laha R. Tailoring plasmonic properties of metal-dielectric nanocomposite thin films. AIP Conference Proceedings. 2017; 1832:050144.

[26] Johnson P, Christy R. Optical Constants of the Noble Metals. Physical Review B. 1972;6(12):4370-4379.

[27] Rosenblatt G, Simkhovich B, Bartal G, Orenstein M. Nonmodal Plasmonics: Controlling the Forced Optical Response of Nanostructures. Physical Review X. 2020;10(1).

[28] Ciesielski A, Skowronski L, Trzcinski M, Szoplik T. Controlling the optical parameters of self-assembled silver films with wetting layers and annealing. Applied Surface Science. 2017; 421:349-356.

[29] Hornyak G, Patrissi C, Martin C, Valmalette J, Dutta J, Hofmann H. Dynamical Maxwell-Garnett optical modeling of nanogold-porous alumina composites: Mie and Kappa influence on absorption maxima. Nanostructured Materials. 1997;9(1-8):575-578.

[30] Campos A, Troc N, Cottancin E, Pellarin M, Weissker H, Lermé J et al. Plasmonic quantum size effects in silver nanoparticles are dominated by interfaces and local environments. Nature Physics. 2018;15(3):275280.

(C) 2021 by the author(s). This work is licensed under a Creative Commons Attribution 4.0 International License (http://creativecommons.org/licenses/by/4.0/). Authors retain copyright of their work, with first publication rights granted to Tech Reviews Ltd. 